between sessions 2 and 3. These sessions were randomised and counterbalanced, and no order effects were observed.

Finally, as Liang \& Ho note, Professor Nutt commented on the two main effects of acute tryptophan depletion. That is, acute tryptophan depletion increased the dopamine response when patients took cocaine, and decreased it when the drug was absent. These opposite effects in the presence $v$. absence of drug might contribute to a core feature of substance misuse: i.e. increased incentive motivational states when drugs and highly salient drug cues are present, and decreases when these stimuli are absent. ${ }^{5}$

1 Linnet J, Peterson E, Doudet DJ, Gjedde A, Møller A. Dopamine release in ventral striatum of pathological gamblers losing money. Acta Psychiatr Scand 2010; 122: 326-33.

2 Linnet J, Peterson E, Gjedde A, Doudet DJ. Inverse association between dopaminergic neurotransmission and lowa Gambling Task performance in pathological gamblers and healthy controls. Scand J Psychol 2011; 52 28-34.

3 Campbell-Meiklejohn D, Wakeley J, Herbert V, Cook J, Scollo P, Ray MK, et al. Serotonin and dopamine play complementary roles in gambling to recover losses. Neuropsychopharmacol 2011; 36: 402-10.

4 Abler B, Grön G, Hartmann A, Metzger C, Walter M. Modulation of frontostriatal interaction aligns with reduced primary reward processing under serotonergic drugs. J Neurosci 2012; 32: 1329-35.

5 Leyton M. Conditioned and sensitized responses to stimulant drugs in humans. Prog Neuropsychopharmacol Biol Psychiatry 2007; 31: 1601-13.

Sylvia M. L. Cox, PhD, Department of Psychiatry and Department of Neurology and Neurosurgery; Kevin F. Casey, MSC, Departments of Psychiatry and Neurology \& Neurosurgery; J. Scott Delaney, MD, Department of Emergency Medicine; \& Neurosurgery; J. Scott Delaney, MD, Department of Emergency Medicine,
Marco Leyton, PhD, Department of Psychiatry and Department of Neurology and Neurosurgery, McGill University, 1033 Pine Avenue West, Montréal, Québec, Canada H3A 1A1. Email: marco.leyton@mcgill.ca

doi: 10.1192/bjp.201.1.73a

\section{Encephalitis and schizophrenia: a matter of words}

The two recent articles ${ }^{1,2}$ on the psychiatric manifestations of antibody-mediated encephalitis are important reminders that a well-informed differential diagnosis has far reaching implications for providing optimal patient care. It is indeed instructive to note that a marked recovery is possible with immunosuppressant therapy. Additionally, the need for close liaison with plasma exchange facilities, gynaecologists, neurologists and immunologists represents a novel departure for many practitioners, we presume. We did, however, have some concerns with the title of the Lennox et al editorial. ${ }^{1}$ Describing the encephalitis as a treatable cause of schizophrenia jarred a little. First, we were concerned that the editorial title could give the impression that other causes of schizophrenia are not treatable. This brings to mind another excellent editorial, by Williams et $a l .^{3}$ They proposed that we should use the term 'neuroleptic resistance' as opposed to treatment resistance when discussing clozapine therapy to avoid therapeutic nihilism. Second, is what is being described schizophrenia or a schizophrenia-like illness? The ICD- $10^{4}$ states that 'schizophrenia should not be diagnosed in the presence of overt brain disease.' As neuroimaging progresses, this stipulation might no longer be tenable. Is it preferable to refer to this type of presentation as a psychosis? However, these are minor quibbles and we will certainly view initial psychotic presentations differently as a consequence of these two important contributions to the psychiatric literature.

1 Lennox BR, Coles AJ, Vincent A. Antibody-mediated encephalitis: a treatable cause of schizophrenia. Br J Psychiatry 2012; 200: 92-4.
2 Barry H, Hardiman O, Healy DG, Keogan M, Moroney J, Molnar PP, et al. AntiNMDA receptor encephalitis: an important differential diagnosis in psychosis. Br J Psychiatry 2011; 199: 508-9.

3 Williams L, Newton G, Roberts K, Finlayson S, Brabbins C. Clozapine-resistant schizophrenia: a positive approach. Br J Psychiatry 2002; 181: 184-7.

4 World Health Organization. The ICD-10 Classification of Mental and Behavioural Disorders: Clinical Descriptions and Diagnostic Guidelines. WHO, 1992.

Kieran O'Loughlin, Senior Registrar, Louth Mental Health Service, Ardee, Co. Louth Ireland. Email: kieran.oloughlin@ucd.ie; Paul Ruge, Registrar, Louth Mental Health Service, Ardee, Co. Louth; MacDara McCauley, Consultant Psychiatrist, Louth Mental Health Service, Ardee, Co. Louth, Ireland

doi: 10.1192/bjp.201.1.74

Authors' reply: We suggest that the evidence shows that although antipsychotics are effective in alleviating some, although often not all, of the symptoms of schizophrenia, there is no evidence that they treat the underlying disorder. The editorial was highlighting the fact that the clinical syndrome of patients with psychosis and $N$-methyl-D-aspartate receptor antibodies is the same as those with schizophrenia, such that most patients with this new disorder have previously received diagnoses of schizophrenia. However, as O'Laughlin et al state, having an identifiable cause invalidates the diagnosis of schizophrenia according to ICD. We agree that a syndrome of psychoses is a better diagnostic construct. This situation is not unique to psychiatry. In epilepsies, despite the rapid advance in discovery of aetiological factors, the diagnosis remains based on the clinical presentation of the seizures.

\section{Declaration of interest}

A.J.C. has received consulting fees, lecture fees and grant support from Genzyme. A.V. and the Nuffield Department of Clinical Neuroscicences/University of Oxford hold patents, receive royalties from Athena Diagnostics, and receive payments for antibody assays.

Belinda R. Lennox, DM, MRCPsych, Cambridgeshire and Peterborough NHS Foundation Trust, and Department of Psychiatry, University of Cambridge, Herchel Smith Building, Cambridge Biomedical Campus, Addenbrooke's Hospital, Cambridge CB2 2QQ, UK. Email: bl224@cam.ac.uk; Alasdair J. Coles, PhD, FRCP, Neurology Unit, Addenbrooke's Hospital, Cambridge; Angela Vincent, FRS, FMedSci, Nuffield Department of Clinical Neurosciences, University of Oxford, John Radcliffe Hospital, Oxford, UK

doi: 10.1192/bjp.201.1.74a

\section{Loss of autism in DSM-5}

I wish to comment on the phrase in the editorial by Tyrer \& Craddock that in DSM-5 'the changes are largely cosmetic.' This is probably correct for most of DSM-5 but not for autism, where a new, narrow definition of autism is proposed. The broader autism phenotype is accepted by professionals in this area of study. A new study has shown that only $60 \%$ of patients meet criteria for DSM-IV autism when they are assessed using the criteria of DSM-5 autism. ${ }^{2}$ A second error in this area in relation to DSM-5 is that an aspect of autism has been split off into a new category called social communication disorder. ICD-11 has not made this error. These changes in DSM-5 in relation to autism are radical and will lead to patients losing their diagnosis and services.

1 Tyrer $\mathrm{P}$, Craddock $\mathrm{N}$. The bicentennial volume of the British Journal of Psychiatry: the winding pathway of mental science. Br J Psychiatry 2012; 200: 1-4. 
2 Yang $Y$, Bai-Lin W, Shen Y. A pilot study on the diagnostic performance of DSM-IV and DSM-V for autism spectrum disorder. N A J Med Sci 2011; 4 $116-23$.

Michael Fitzgerald, Professor of Child Psychiatry, Trinity College Dublin, Ireland. Email: fitzi@iol.ie

doi: $10.1192 / \mathrm{bjp} .201 .1 .74 \mathrm{~b}$

\section{The Department of Health and the Equality} Act 2010

In their otherwise excellent review of the Equality Act 2010 and mental health, ${ }^{1}$ the authors did not highlight how the Department of Health currently discriminates against people with mental health problems.

The National Health Service (NHS) constitution has incorporated the Equality Act in terms of access to NHS care, including on the grounds of disability. However, a fundamental right of the constitution is that of choice. Section 2a states 'You have the right to make choices about your NHS care and to information to support these choices. The options available to you will develop over time and depend on your individual needs. ${ }^{2}$

Since April 2009, patients have had a right to choose the service that provides their treatment when they are referred for their first out-patient appointment with a consultant-led team. Patients can review outcome data, specialist expertise and user feedback for a service, discuss it with their general practitioner, and be referred for an elective medical or surgical problem to any NHS consultant-led service across the country. However, the Department of Health excludes patients detained under the Mental Health Act 1983, military personnel and prisoners. It also excludes services where speed of access to diagnosis and treatment is important, for example emergency admissions and maternity services. However, under this clause it also excludes elective mental health services. This appears to be discriminatory under the Equality Act for people with mental health problems who are disabled by their disorder. So anyone with a mental disorder who is disabled and has had treatment locally cannot by right obtain a referral to a specialist mental health service. Most National Institute for Health and Clinical Excellence guidelines on mental disorders envisage stepped care. Where treatment has failed, the next step is onward referral to more intensively delivered cognitive-behavioural therapy (e.g. more frequent, longer sessions with more experienced therapists) or to specialised pharmacological advice that may not be available from a local community mental health team or psychology service.

Patients with mental disorders who are disabled therefore have the right to choose where they have treatment for their cancer, for example, but not for their mental disorder. Access depends entirely on the vagaries of local funding panels. The legal right to choice of elective care should be extended to mental health services, or withdrawn from surgery and medicine. The present discrimination is unconscionable.

1 Lockwood G, Henderson C, Thornicroft G. The Equality Act 2010 and mental health. Br J Psychiatry 2012; 200: 182-3.

2 Department of Health. The Handbook to the NHS Constitution. Department of Health, 2012.

David Veale, FRCPsych, Consultant Psychiatrist, South London and Maudsley NHS Foundation Trust, and Institute of Psychiatry, King's College London, UK. Email: David.Veale@kcl.ac.uk

doi: 10.1192/bjp.201.1.75

\section{Monitoring cardiometabolic risk in schizophrenia}

I commend De Hert et $a l^{1}$ for their attempt to clarify appropriate monitoring for cardiometabolic risk in schizophrenia. I agree that cardiometabolic risk is one important consideration for these patients.

I note that their findings included generally low scores for the rigour of existing guidelines and a lack of evidence of long-term patient outcomes. It is perhaps a little surprising then that they nevertheless make recommendations on what appears to be less than robust evidence.

I have previously expressed concerns that cardiometabolic screening programmes of this type are unevaluated and that the benefits are unknown, as are the risks, which seem to have received little attention. ${ }^{2}$

The authors quite rightly highlight that guidelines can be biased because of lack of scientific evidence, but the evidence they present to support their protocol appears to fall well short of the levels of evidence recommended for interventions. ${ }^{3}$ I can find no evidence that patients will benefit from such a protocol, and none that they will not be harmed.

I also note that their suggested protocol differs from National Institute for Health and Clinical Excellence (NICE) quality and outcomes framework standards for mental illness (www.nice. org.uk/aboutnice/qof/indicators.jsp) and NICE guidelines for lipid modification, both of which recommend primary preventive screening for patients aged over $40 .^{4}$

I wish to support the notion that interventions should be evaluated before implementation. ${ }^{3}$

1 De Hert M, Vancampfort D, Correll CU, Mercken V, Peuskens J, Sweers K, et al. Guidelines for screening and monitoring of cardiometabolic risk in schizophrenia: systematic evaluation. Br J Psychiatry 2011; 199: 99-105.

2 Reed PF. Let's target screening more effectively. Psychiatrist 2010; 34 $540-1$.

3 Oxford Centre for Evidence-Based Medicine. CEBM (Centre for EvidenceBased Medicine) Levels of Evidence. CEBM, 2012 (http://www.cebm.net/ index.aspx?0=5653)

4 National Institute for Health and Clinical Excellence. Lipid Modification: Cardiovascular Risk Assessment and the Modification of Blood Lipids for the Primary and Secondary Prevention of Cardiovascular Disease (Clinical Guideline 67). NICE, 2008.

Paul F. Reed, Consultant Psychiatrist, Lancashire Care NHS Foundation Trust, UK. Email: paul.reed@lancashirecare.nhs.uk

doi: 10.1192/bjp.201.1.75a

Physical care of people with severe mental illness is an important clinical issue, as the potential health benefits of cardiovascular disease prevention for the general population are astonishing. Each year, cardiovascular disease kills about 20 million people, including 10 million prematurely (before the age of 65 years) and inflicts high morbidity, disability and socioeconomic costs. ${ }^{1}$ This problem is more pronounced in schizophrenia, with standardised mortality rates (SMRs) of 2.7 for diabetes and 2.3 for cardiovascular disease. ${ }^{2}$

Cardiovascular mortality increased in schizophrenia from 1976 to 1995 , with the greatest increase in SMR in men from 1991 to $1996 .{ }^{3}$

In the current climate of austerity in the National Health Service and internationally, it is interesting to know that in high-income countries, preventing or postponing 100 cases has been reported as saving about US\$1 million ( $£ 0.6$ million, $€ 0.7$ million). ${ }^{4}$ 